\title{
DESENVOLVIMENTO DO PROJETO CONCEITUAL DE LAYOUT PARA UMA EMPRESA DE ARTEFATOS DE CONCRETO
}

\section{DEVELOPMENT OF THE CONCEPTUAL DESIGN OF LAYOUT FOR A COMPANY OF CONCRETE ARTIFACTS}

\section{R. MOURA ${ }^{1, *}$, L. OLIVEIRA ${ }^{2}$, G. C. OROSKI ${ }^{3}$, M. GAMBETA ${ }^{4}$ e T. L. SOARES $^{5}$}

${ }^{1}$ Federal Institute of Santa Catarina, Department of Mechanical manufacturing, Jaraguá do Sul, SC, Brazil

${ }^{2}$ State University of Santa Catarina, Production Engineering Department, Joinville, SC, Brazil

${ }^{3}$ State University of Santa Catarina, Production Engineering Department, Joinville, SC, Brazil

${ }^{4}$ State University of Santa Catarina, Production Engineering Department, Joinville, SC, Brazil

${ }^{5}$ State University of Santa Catarina, Production Engineering Department, Joinville, SC, Brazil

${ }^{*}$ Corresponding author. Federal Institute of Santa Catarina, Department of Mechanical manufacturing, Jaraguá do Sul, SC, Brazil, Phone: +55 47 3276-9600

e-mail address: cassiano.moura@ifsc.edu.br (C. R. Moura).

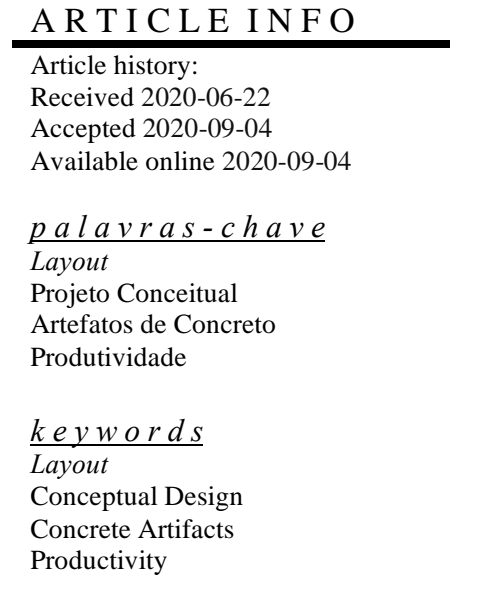

\section{R E S U M O}

Um Layout bem desenvolvido pode contribuir positivamente com os resultados das organizações principalmente no que diz respeito às questões de aumento de produtividade e otimização de espaço físico. Diante disso este artigo tem como objetivo geral desenvolver o projeto conceitual de um layout em uma empresa de Artefatos de Concreto, visando a melhor utilização do espaço fabril. Para isto são utilizadas ferramentas e técnicas de apoio para complementar a realização deste projeto. A sequência do projeto é definida de acordo com: definição das unidades de planejamento de espaços (UPE), resumo das UPE's, diagrama de afinidades entre UPE's, diagrama de configuração, realização de layouts, análises de movimentações de pessoas, materiais e informações para cada layout proposto e diagrama de uso de espaço para os layouts propostos, além da matriz de seleção das propostas de layout. Como resultado são apresentadas 3 propostas de layout que são avaliados através de critérios como movimentação de materiais, adequação do ambiente, custo e acessibilidade. Por fim um é selecionado para a continuação do projeto detalhado de layout a ser aplicado na organização.

A B S T R A C T

A well-developed layout can positively contribute to the results of organizations, especially with regard to issues of increased productivity and optimization of physical space. Given this, this article has the general objective to develop the conceptual design of a layout in a Concrete Artifacts company, aiming at the best use of the manufacturing space. For this, tools and support techniques are used to complement the realization of this project. The project sequence is defined according to: Definition of Space Planning Units (UPE), Summary of UPE's, Diagram of Affinities between UPE's, Configuration Diagram, Layouts, Analysis of movements of people, materials and information for each proposed layout and Space Usage Diagram for the proposed layouts, in addition to the selection matrix of the layout proposals. As a result, 3 layout proposals are presented, which are evaluated using criteria such as movement of materials, suitability of the environment, cost and accessibility. Finally, one is selected for the continuation of the detailed layout design to be applied in the organization. 


\section{INTRODUÇÃO}

$\mathrm{Na}$ busca pelo aumento da eficiência competitiva, as organizações procuram desenvolver inúmeras ações para alcançar esse objetivo, entre elas trabalhar com layouts mais organizados e eficientes, porém isso normalmente requer alterações bruscas ou novos galpões e imóveis, o que consequentemente resulta em valores maiores de investimento.

Estudos apontam que melhorias no processo fabril decorrentes de alterações de layout podem aumentar a eficiência produtiva das empresas. Isso ocorre devido à redução de desperdícios alinhado ao balanceamento dos setores produtivos (MOURA, 2019; OLIVEIRA et al., 2017).

Bonecher et al (2020) realizou um levantamento da situação atual e das necessidades de uma empresa, para simular diferentes cenários de layout analisando suas vantagens e desvantagens utilizando dados quantitativos e considerando suas restrições físicas, na busca de sanar as dificuldades de capacidade enfrentadas pela organização.

Para Neumann e Scalice (2015) a realização do projeto de layout fabril se diferencia nos aspectos de complexidade e novidade. No caso de alterações no layout atual para organizações de pequeno a médio porte é recomendado à utilização do modelo de projeto fabril proposto por eles, porém com simplificações, pois parte do levantamento das informações já ocorrem durante a utilização do layout ou derivadas do projeto anterior, por se tratar de organizações de menor porte é recomendada a realização do reprojeto do layout completo da organização, porém alterações de menor porte também são possíveis.

Um aumento na produtividade de um setor pode resultar em $15 \%$ de ganhos, sem que haja necessidade de investimento em novos equipamentos, somente com modificações no layout através de métodos racionais e detalhados do processo (MOREIRA, 2004).

Para realização do projeto de layout é importante criar temporariamente uma equipe multidisciplinar que possua grande conhecimento dos produtos e processos a serem introduzidos no novo layout, caso a empresa não possua pessoas qualificadas para a alteração do layout da organização é recomendada a contratação de especialistas, pois a realização de um layout inadequado pode gerar gastos desnecessários para organização (NEUMANN E SCALICE, 2015).

Segundo Gurgel (2008) um bom layout pode ser caracterizado por: planejamento do modelo dos fluxos de materiais, fluxo de atividades orientado para o mercado, layout com elevado grau de flexibilidade, layout com possibilidade de expansão, área de armazenamento adequada, posicionamento dos operadores sem que seja necessários deslocamentos.

\section{METODOLOGIA}

A pesquisa adotada neste trabalho pode ser classificada como qualitativa, que de acordo com Flick (2009) tem por objetivo encontrar o que há de novo, para posteriormente iniciar o desenvolvimento de teorias empiricamente fundamentadas. $\mathrm{O}$ fluxo da pesquisa contou com dados primários e secundários, e se caracterizou por ser exploratória. A figura 1 apresenta o fluxo metodológico adotado no trabalho, onde se pode observar a sequência lógica para o desenvolvimento do projeto conceitual do layout.

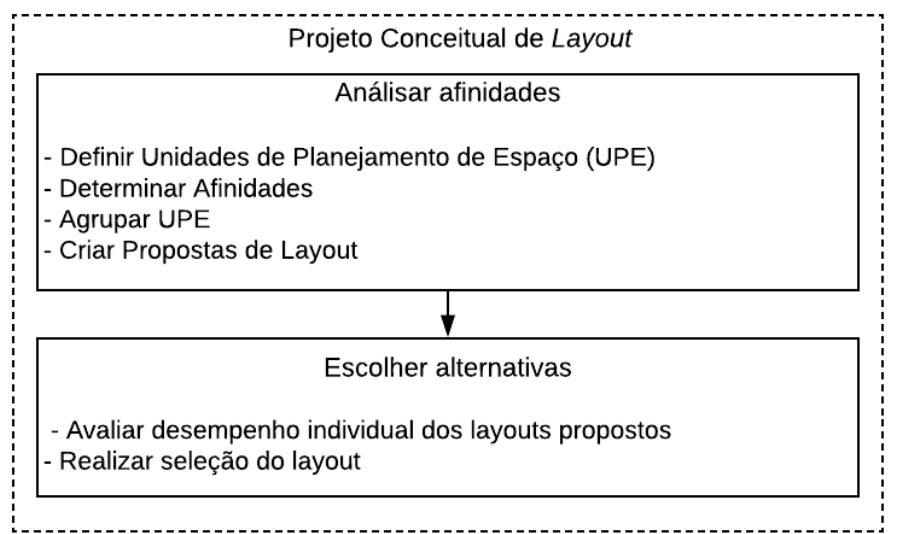

Figura 1 - Fluxo metodológico adotado neste trabalho

O Projeto conceitual para realização do layout tem como objetivo principal desdobrar em várias alternativas de layout fabril o Planejamento tático fabril, para que elas sejam comparadas entre si e por fim seja determinada a mais atrativa para a organização. Para a realização dessa fase, devem ser cumpridas as seguintes etapas: Analisar afinidades e Escolher Alternativas.

As atividades de analisar afinidades consistem em: definir as Unidades de Planejamento de Espaço (UPE); determinar afinidades; agrupar upe; criar propostas de layout. As atividades de escolher alternativas consistem em: avaliar desempenho individual dos layouts propostos; realizar seleção do layout.

\subsection{Analisar afinidades}

A fase de Analisar Afinidades tem como objetivo transformar as informações adquiridas no Projeto informacional para realização do layout fabril e por final gerar um conjunto de propostas de layout dentro do esperado pela organização. As atividades de Analisar Afinidades consistem em: Definir Unidades de Planejamento de Espaço (UPE); Determinar Afinidades; Agrupar UPE; Criar Propostas de Layout. Estas são descritas a seguir.

\section{a) Definir Unidades de Planejamento de Espaço (UPE)}

Nesta etapa são definidas quais as unidades que farão parte do planejamento do Layout, a tabela 1 apresenta quais as áreas que compõem a empresa de Artefatos de cimento e que estão em estudo neste trabalho. 
Tabela 1 - Unidades de Planejamento de Espaços da Empresa de Artefatos de Concreto.

\begin{tabular}{ll}
\hline \multicolumn{1}{c}{$\begin{array}{c}\text { Unidades de Planejamento do Espaços da Empresa de } \\
\text { Artefatos de Concreto }\end{array}$} \\
\hline 1 & Recepção e Administrativo \\
\hline 2 & Banheiros \\
\hline 3 & Copa \\
\hline 4 & Oficina de manutenção \\
\hline 5 & Almoxarifado \\
\hline 6 & Área produtiva \\
\hline 7 & Estoque de produtos acabados \\
\hline 8 & Estoque de produtos de revenda \\
\hline 9 & Estoque de matéria prima \\
\hline
\end{tabular}

\begin{tabular}{ll}
10 & Estacionamento Clientes \\
\hline 11 & Garagem para caminhões Grandes \\
\hline 12 & Garagem para caminhão pequeno \\
\hline 13 & Garagem para empilhadeira e trator concha \\
\hline 14 & Área de carregamento de caminhão \\
\hline
\end{tabular}

Após a definição das UPE’s, estas são agrupadas e identificadas como Resumo de UPE conforme mostra a Tabela 2, na qual são observadas suas características bem como realizadas as inclusões e/ou exclusões em algumas UPE's. Também é registrada neste momento a definição de cada área onde é expressa sua finalidade.

Tabela 2 - Resumo da Unidades de Planejamento de Espaços na empresa de Artefatos de Concreto.

\begin{tabular}{|c|c|c|c|c|c|c|c|c|c|c|c|c|}
\hline $\mathbf{N}^{\mathbf{o}}$ & 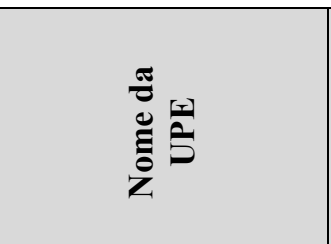 & 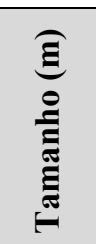 & 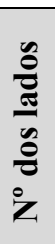 & 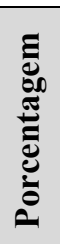 & 异 & 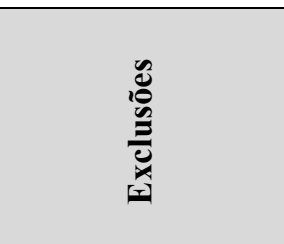 & 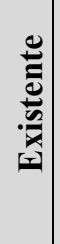 & 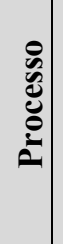 & | & 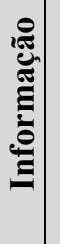 & 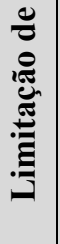 & 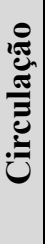 \\
\hline 1 & $\begin{array}{c}\text { Recepção e } \\
\text { Administrativo }\end{array}$ & $4 \times 9$ & 2 & 30 & Vendas, escritório e recepção & $\begin{array}{c}\text { Copa para } \\
\text { funcionários }\end{array}$ & $\mathrm{X}$ & & $\mathrm{X}$ & & & \\
\hline 2 & Banheiros & $4 \times 4$ & 1 & 10 & $\begin{array}{l}2 \text { banheiros com chuveiro } \\
\text { (Feminino/Masculino) }\end{array}$ & & & & & $\mathrm{X}$ & & \\
\hline 3 & Copa & $4 \times 4$ & 1 & 10 & $\begin{array}{c}\text { Cafeteria, Micro-ondas, Mesa, } \\
\text { Geladeira, Armário }\end{array}$ & & & & & $\mathrm{X}$ & & \\
\hline 4 & $\begin{array}{l}\text { Oficina de } \\
\text { manutenção }\end{array}$ & $4 \times 6$ & 1 & 20 & $\begin{array}{l}\text { Ferramentas e insumos de } \\
\text { manutenção }\end{array}$ & $\begin{array}{c}\text { Ferramentas de } \\
\text { trabalho }\end{array}$ & $\mathrm{X}$ & $\mathrm{X}$ & & & & \\
\hline 5 & Almoxarifado & $4 \times 6$ & 1 & 20 & $\begin{array}{l}\text { Ferramentas de trabalho e } \\
\text { insumos de processo }\end{array}$ & Matéria-prima & $\mathrm{X}$ & $\mathrm{X}$ & & $\mathrm{X}$ & & \\
\hline 6 & Área produtiva & $10 \times 9$ & 4 & 30 & $\begin{array}{l}\text { Betoneira, mesa vibratória, área } \\
\text { de secagem }\end{array}$ & Matéria-prima & $\mathrm{X}$ & $\mathrm{X}$ & & $\mathrm{X}$ & & \\
\hline 7 & $\begin{array}{l}\text { Estoque de produtos } \\
\text { acabados }\end{array}$ & $20 \times 5$ & 2 & 40 & $\begin{array}{c}\text { Produtos acabados } \\
\text { manufaturados na empresa }\end{array}$ & $\begin{array}{l}\text { Produtos de } \\
\text { revenda }\end{array}$ & $\mathrm{X}$ & & & & $\mathrm{X}$ & $\mathrm{X}$ \\
\hline 8 & $\begin{array}{c}\text { Estoque de produtos } \\
\text { de revenda }\end{array}$ & $15 \times 5$ & 3 & 40 & Produtos de revenda & $\begin{array}{l}\text { Produtos acabados } \\
\text { manufaturados }\end{array}$ & $\mathrm{X}$ & & & & $\mathrm{X}$ & $\mathrm{X}$ \\
\hline 9 & $\begin{array}{c}\text { Estoque de matéria } \\
\text { prima } \\
\end{array}$ & $35 \times 5$ & 1 & 40 & Matéria-prima & $\begin{array}{c}\text { Produtos-acabados } \\
\text { e insumos } \\
\end{array}$ & $X$ & & & & $\mathrm{X}$ & $\mathrm{X}$ \\
\hline 10 & $\begin{array}{l}\text { Estacionamento } \\
\text { Clientes }\end{array}$ & $10 \times 5$ & 1 & 30 & $\begin{array}{c}4 \text { vagas de estacionamento para } \\
\text { clientes }\end{array}$ & & & & $X$ & & & $\mathrm{X}$ \\
\hline 11 & $\begin{array}{c}\text { Garagem para } \\
\text { caminhões Grandes }\end{array}$ & $7 \times 12$ & 1 & 30 & $\begin{array}{c}\text { Garagem coberta para } 3 \\
\text { caminhões grandes }\end{array}$ & & & & & & $X$ & \\
\hline 12 & $\begin{array}{c}\text { Garagem para } \\
\text { caminhão pequeno }\end{array}$ & $3 \times 7$ & 1 & 30 & $\begin{array}{l}\text { Garagem coberta para } 1 \\
\text { caminhão pequeno }\end{array}$ & & & & & & $X$ & \\
\hline 13 & $\begin{array}{c}\text { Garagem para } \\
\text { empilhadeira e trator } \\
\text { concha } \\
\end{array}$ & $3 \times 7$ & 1 & 20 & $\begin{array}{l}\text { Garagem coberta para } 1 \\
\text { empilhadeira pequena e } 1 \text { trator } \\
\text { concha grande }\end{array}$ & & & & & & $X$ & \\
\hline 14 & $\begin{array}{c}\text { Área de carregamento } \\
\text { de caminhão }\end{array}$ & $7 \times 4$ & 4 & 30 & $\begin{array}{l}\text { Área livre de qualquer } \\
\text { interferência }\end{array}$ & & & $X$ & & & & $\mathrm{X}$ \\
\hline
\end{tabular}

b) Determinar Afinidades

Para elaborar o Diagrama de Afinidades foi realizado o cruzamento das 14 UPEôs para que fosse analisada as relações entre elas. No caso de haver relação entre 2 UPEô, deve analisado qual o grau desta relação, bem como se há movimentações de pessoas ou materiais neste fluxo. As convenções de afinidade a serem analisadas devem seguir a convenção indicada na Tabela 3 . 
Tabela 3 - Convenções de afinidades.

\begin{tabular}{cccc}
\hline \multicolumn{4}{c}{ Convenções de afinidades } \\
\hline Descrição & Vogal & Escala & Cor \\
Absoluta & $\mathrm{A}$ & 4 & Vermelho \\
Excepcional & $\mathrm{E}$ & 3 & Amarelo \\
Importante & $\mathrm{I}$ & 2 & Verde \\
Ordinária & $\mathrm{O}$ & 1 & Azul \\
Sem importância & $\mathrm{U}$ & 0 & - \\
\hline
\end{tabular}

\begin{tabular}{cccc}
\hline Distante & $X$ & -1 & Preto \\
\hline
\end{tabular}

Fonte: adaptado de LEE (1998).

Na Tabela 4 pode ser observado o diagrama de afinidades aplicado no projeto de layout da empresa em estudo, nesta são relacionadas as afinidades das 14 UPEôs.

Tabela 4 - Diagrama de afinidades para o projeto de layout na empresa de Artefatos de Concreto.

\begin{tabular}{|c|c|c|c|c|c|c|c|c|c|c|c|c|c|c|c|}
\hline & & 1 & 2 & 3 & 4 & 5 & 6 & 7 & 8 & 9 & 10 & 11 & 12 & 13 & 14 \\
\hline & UPES & 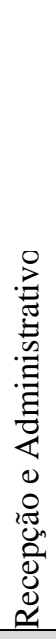 & 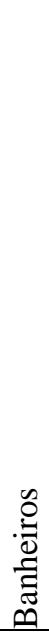 & ठ̊. & 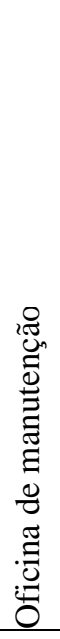 & 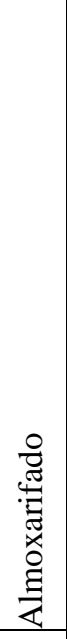 & 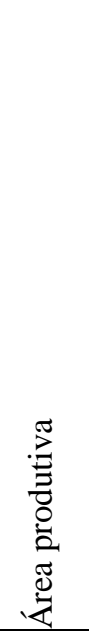 & 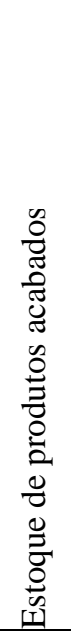 & $\begin{array}{l}\text { चु } \\
0 \\
0 \\
0 \\
0 \\
0 \\
0 \\
0 \\
0 \\
0 \\
0 \\
0 \\
0 \\
0 \\
0 \\
0 \\
0 \\
0 \\
0\end{array}$ & 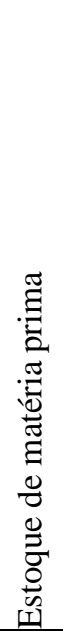 & 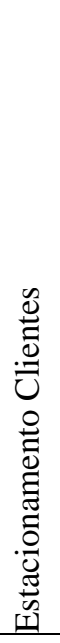 & 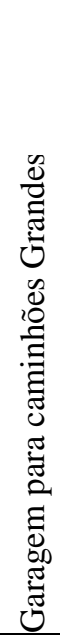 & 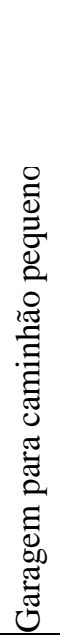 & 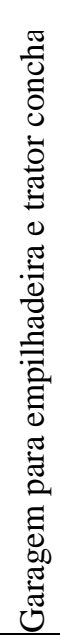 & 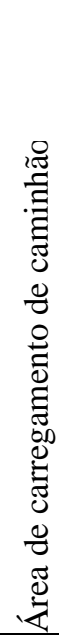 \\
\hline 1 & Recepção e Administrativo & & $\begin{array}{l}\mathrm{E} \\
1\end{array}$ & $\mathrm{U}$ & $\mathrm{U}$ & $\mathrm{U}$ & $\begin{array}{c}\mathrm{X} \\
1 \\
\end{array}$ & $\begin{array}{l}\mathrm{I} \\
1 \\
\end{array}$ & $\begin{array}{l}\mathrm{I} \\
1 \\
\end{array}$ & $\mathrm{U}$ & $\begin{array}{c}\mathrm{E} \\
1\end{array}$ & $\mathrm{U}$ & $\mathrm{U}$ & $\mathrm{U}$ & $\mathrm{U}$ \\
\hline 2 & Banheiros & $\begin{array}{c}\mathrm{E} \\
1 \\
\end{array}$ & & $\begin{array}{c}\mathrm{E} \\
1 \\
\end{array}$ & $\mathrm{U}$ & $\mathrm{U}$ & $\mathrm{U}$ & $\mathrm{U}$ & $\mathrm{U}$ & $\mathrm{U}$ & $\mathrm{U}$ & $\mathrm{U}$ & $\mathrm{U}$ & $\mathrm{U}$ & $\mathrm{U}$ \\
\hline 3 & Copa & $\mathrm{U}$ & $\begin{array}{l}\mathrm{E} \\
1 \\
\end{array}$ & & $\mathrm{U}$ & $\mathrm{U}$ & $\mathrm{U}$ & $\mathrm{U}$ & $\mathrm{U}$ & $\mathrm{U}$ & $\mathrm{U}$ & $\mathrm{U}$ & $\mathrm{U}$ & $\mathrm{U}$ & $\mathrm{U}$ \\
\hline 4 & Oficina de manutenção & $\mathrm{U}$ & $\mathrm{U}$ & $\mathrm{U}$ & & $\begin{array}{c}\mathrm{U} \\
1,2 \\
\end{array}$ & I 1,2 & $\mathrm{U}$ & $\mathrm{U}$ & $\mathrm{U}$ & $\mathrm{U}$ & $\mathrm{U}$ & $\mathrm{U}$ & $\mathrm{U}$ & $\mathrm{U}$ \\
\hline 5 & Almoxarifado & $\mathrm{U}$ & $\mathrm{U}$ & $\mathrm{U}$ & $\begin{array}{c}\mathrm{U} \\
1,2\end{array}$ & & $\begin{array}{c}\mathrm{A} \\
1,2\end{array}$ & $\mathrm{U}$ & $\mathrm{U}$ & $\mathrm{U}$ & $\mathrm{U}$ & $\mathrm{U}$ & $\mathrm{U}$ & $\mathrm{U}$ & $\mathrm{U}$ \\
\hline 6 & Área produtiva & $\begin{array}{c}\mathrm{X} \\
1 \\
\end{array}$ & $\mathrm{U}$ & $\mathrm{U}$ & $\begin{array}{c}\mathrm{I} \\
1,2 \\
\end{array}$ & $\begin{array}{c}\mathrm{A} \\
1,2 \\
\end{array}$ & & $\begin{array}{c}\mathrm{A} \\
1,2\end{array}$ & $\mathrm{U}$ & $\begin{array}{c}\mathrm{A} \\
1,2\end{array}$ & $\mathrm{U}$ & $\mathrm{U}$ & $\mathrm{U}$ & $\mathrm{U}$ & $\begin{array}{c}\mathrm{O} \\
1 \\
\end{array}$ \\
\hline 7 & Estoque de produtos acabados & $\begin{array}{l}\mathrm{I} \\
1 \\
\end{array}$ & $\mathrm{U}$ & $\mathrm{U}$ & $\mathrm{U}$ & $\mathrm{U}$ & $\begin{array}{c}\mathrm{A} \\
1,2 \\
\end{array}$ & & $\mathrm{U}$ & $\mathrm{U}$ & $\mathrm{U}$ & $\mathrm{U}$ & $\mathrm{U}$ & $\mathrm{U}$ & $\begin{array}{c}\mathrm{A} \\
1,2 \\
\end{array}$ \\
\hline 8 & $\begin{array}{l}\text { Estoque de produtos de } \\
\text { revenda }\end{array}$ & $\begin{array}{l}\mathrm{I} \\
1\end{array}$ & $\mathrm{U}$ & $\mathrm{U}$ & $\mathrm{U}$ & $\mathrm{U}$ & $\mathrm{U}$ & $\mathrm{U}$ & & A & $\mathrm{U}$ & $\mathrm{U}$ & $\mathrm{U}$ & $\mathrm{U}$ & $\begin{array}{c}\mathrm{A} \\
1,2 \\
\end{array}$ \\
\hline 9 & Estoque de matéria prima & $\mathrm{U}$ & $\mathrm{U}$ & $\mathrm{U}$ & $\mathrm{U}$ & $\mathrm{U}$ & $\begin{array}{c}\mathrm{A} \\
1,2 \\
\end{array}$ & $\mathrm{U}$ & $\mathrm{U}$ & & $\mathrm{U}$ & $\mathrm{U}$ & $\mathrm{U}$ & $\mathrm{U}$ & $\begin{array}{l}\mathrm{E} \\
2 \\
\end{array}$ \\
\hline 10 & Estacionamento Clientes & $\begin{array}{c}\mathrm{E} \\
1\end{array}$ & $\mathrm{U}$ & $\mathrm{U}$ & $\mathrm{U}$ & $\mathrm{U}$ & $\mathrm{U}$ & $\mathrm{U}$ & $\mathrm{U}$ & $\mathrm{U}$ & & $\mathrm{U}$ & $\mathrm{U}$ & $\mathrm{U}$ & $\mathrm{U}$ \\
\hline 11 & $\begin{array}{l}\text { Garagem para caminhões } \\
\text { Grandes }\end{array}$ & $\mathrm{U}$ & $\mathrm{U}$ & $\mathrm{U}$ & $\mathrm{U}$ & $\mathrm{U}$ & $\mathrm{U}$ & $\mathrm{U}$ & $\mathrm{U}$ & $\mathrm{U}$ & $\mathrm{U}$ & & $\mathrm{U}$ & $\mathrm{U}$ & $\begin{array}{l}\mathrm{O} \\
2 \\
\end{array}$ \\
\hline 12 & $\begin{array}{l}\text { Garagem para caminhão } \\
\text { pequeno }\end{array}$ & $\mathrm{U}$ & $\mathrm{U}$ & $\mathrm{U}$ & $\mathrm{U}$ & $\mathrm{U}$ & $\mathrm{U}$ & $\mathrm{U}$ & $\mathrm{U}$ & $\mathrm{U}$ & $\mathrm{U}$ & $\mathrm{U}$ & & $\mathrm{U}$ & $\begin{array}{l}\mathrm{O} \\
2 \\
\end{array}$ \\
\hline 13 & $\begin{array}{l}\text { Garagem para empilhadeira e } \\
\text { trator concha }\end{array}$ & $\mathrm{U}$ & $\mathrm{U}$ & $\mathrm{U}$ & $\mathrm{U}$ & $\mathrm{U}$ & $\mathrm{U}$ & $\mathrm{U}$ & $\mathrm{U}$ & $\mathrm{U}$ & $\mathrm{U}$ & $\mathrm{U}$ & $\mathrm{U}$ & & $\begin{array}{l}\mathrm{O} \\
2\end{array}$ \\
\hline 14 & $\begin{array}{l}\text { Área de carregamento de } \\
\text { caminhão }\end{array}$ & $\mathrm{U}$ & $\mathrm{U}$ & $\mathrm{U}$ & $\mathrm{U}$ & $\mathrm{U}$ & $\begin{array}{l}\mathrm{O} \\
1\end{array}$ & $\begin{array}{c}\mathrm{A} \\
1,2\end{array}$ & $\begin{array}{l}\mathrm{A} \\
1,2 \\
\end{array}$ & $\begin{array}{l}\mathrm{E} \\
2 \\
\end{array}$ & $\mathrm{U}$ & $\begin{array}{l}\mathrm{O} \\
2 \\
\end{array}$ & $\begin{array}{l}\mathrm{O} \\
2 \\
\end{array}$ & $\begin{array}{l}\mathrm{O} \\
2 \\
\end{array}$ & \\
\hline
\end{tabular}


c) Agrupar UPE

Nesta etapa são avaliadas as possibilidades de combinação entre as UPE definidas no projeto de layout, para isso é elaborado o Diagrama de Configuração para identificar um possível agrupamento entre as UPE, conforme visualizado na Figura 2, onde se pode observar que os fluxos de materiais pessoas e informações são distintos por cores. Os principais fluxos são, fluxo de pessoas (linha azul); fluxo de materiais (linha vermelha) e fluxo de informações (linha verde).

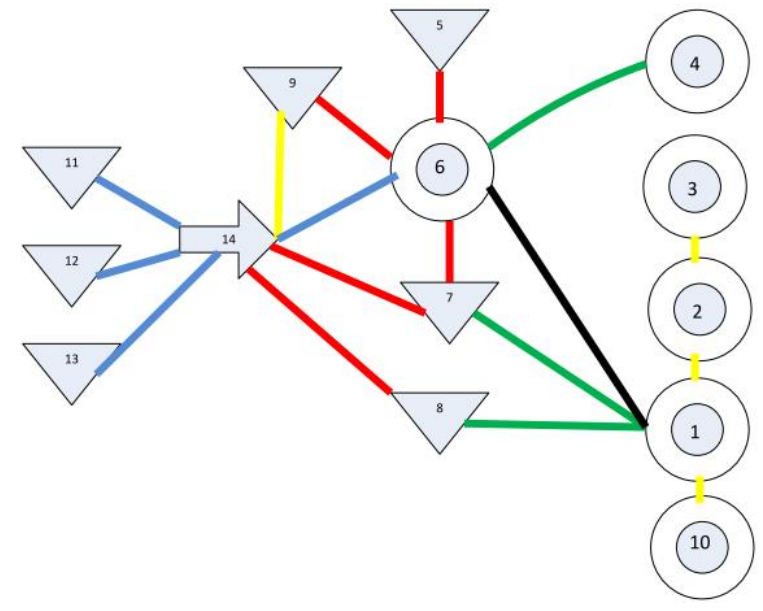

Figura 2 - Diagrama de Configuração na empresa de Artefatos de Concreto.

\section{DESENVOLVIMENTO}

Neste capítulo serão apresentados os resultados do processo de desenvolvimento dos layouts. Estes estão divididos em 3 propostas.

\subsection{Desenvolvimento da proposta 1}

Esta proposta corresponde ao que foi estabelecido no diagrama de afinidades, procura-se diminuir a distância entre os Estoques de Matéria-Prima, a Área Produtiva e o Estoque de Produtos Acabados, conforme mostra a Figura 3.

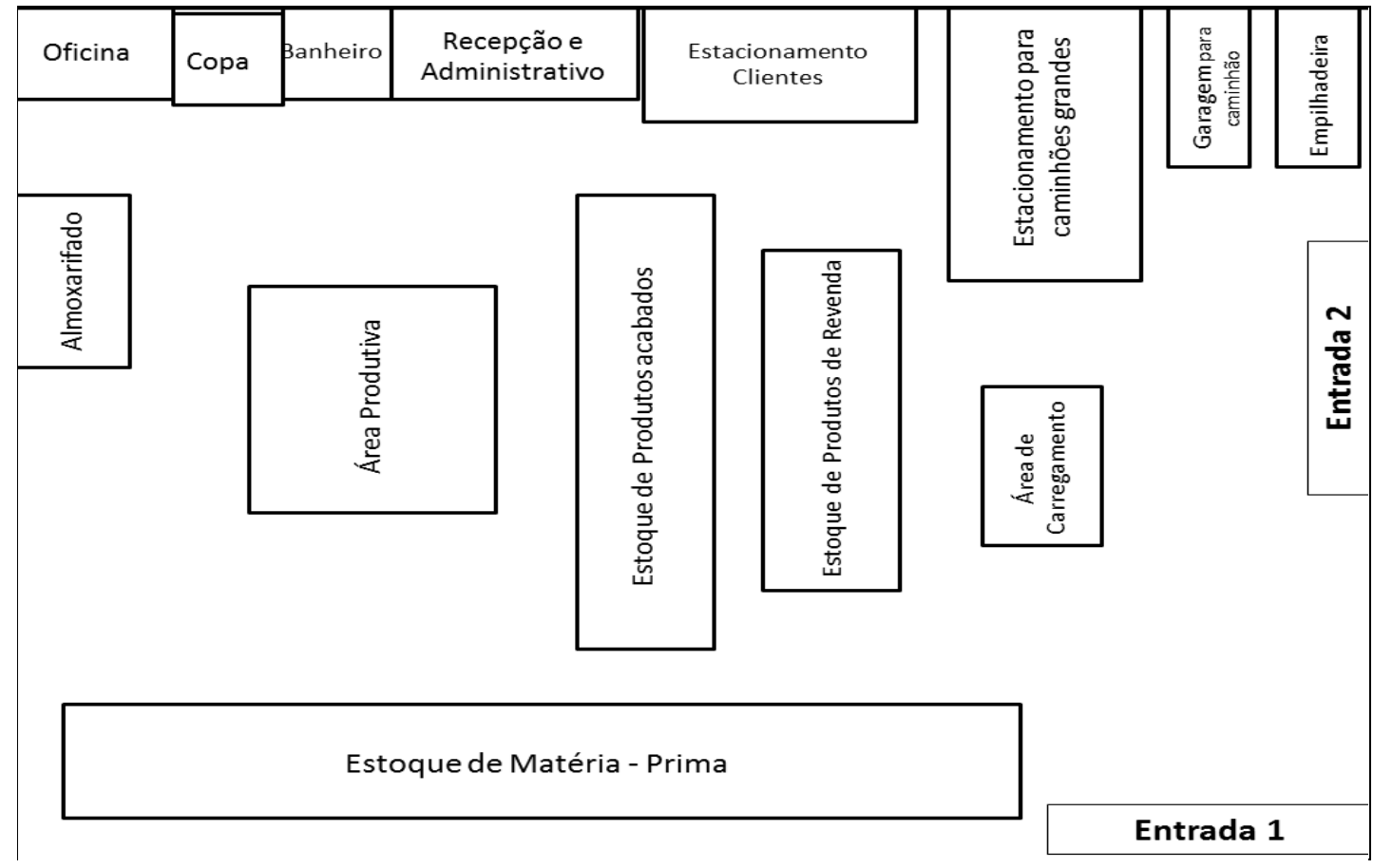

Figura 3 - Diagrama de Configuração na empresa de Artefatos de Concreto. 


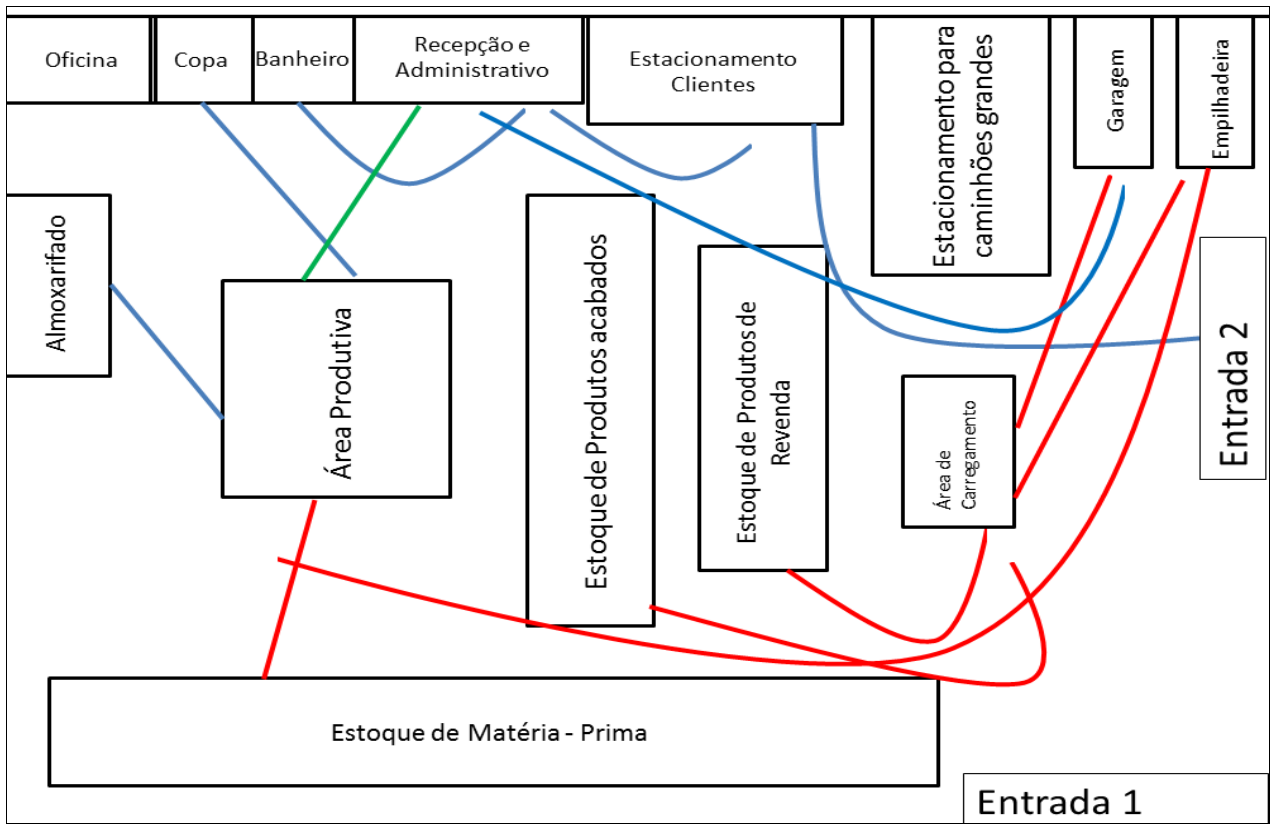

Figura 4 - Análises do layout referente a Proposta 1 de Layout para a empresa de Artefatos de Concreto.

Na figura 4 é apresentado o fluxo de pessoas (linha azul), o fluxo de materiais (linha vermelha) e o fluxo de informações (linha verde), além do espaço que será utilizado para a realização das operações e movimentações na proposta de layout 1.

\subsection{Desenvolvimento da proposta 2}

Esta proposta corresponde melhor arranjo das UPES, conforme melhor distribuição dentro do terreno; Incluída uma saída do terreno exclusiva para caminhões carregados; Estacionamento exclusivo para clientes ao lado da recepção; Recepção próxima da rua para que as atividades e atendimento a clientes não interfira nas atividades de produção e movimentação dentro do terreno; Nessa proposta cerca de $70 \%$ do terreno seria utilizada para as atividades e $30 \%$ permaneceriam como área sem uso, as mesmas podendo ser utilizadas futuramente para expansão ou inclusão de novas UPES. Ponto negativo desta proposta é que demanda muito investimento, pois será necessário derrubar o barracão existente e construir a infraestrutura que cada UPE demande isoladamente. Na figura 5 está indicada a proposta de layout $2 \mathrm{e}$ na figura 6 é apresentado o fluxo de pessoas (linha azul), o fluxo de materiais (linha vermelha) e o fluxo de informações (linha verde), além do espaço que será utilizado para a realização das operações e movimentações na proposta de layout 2.

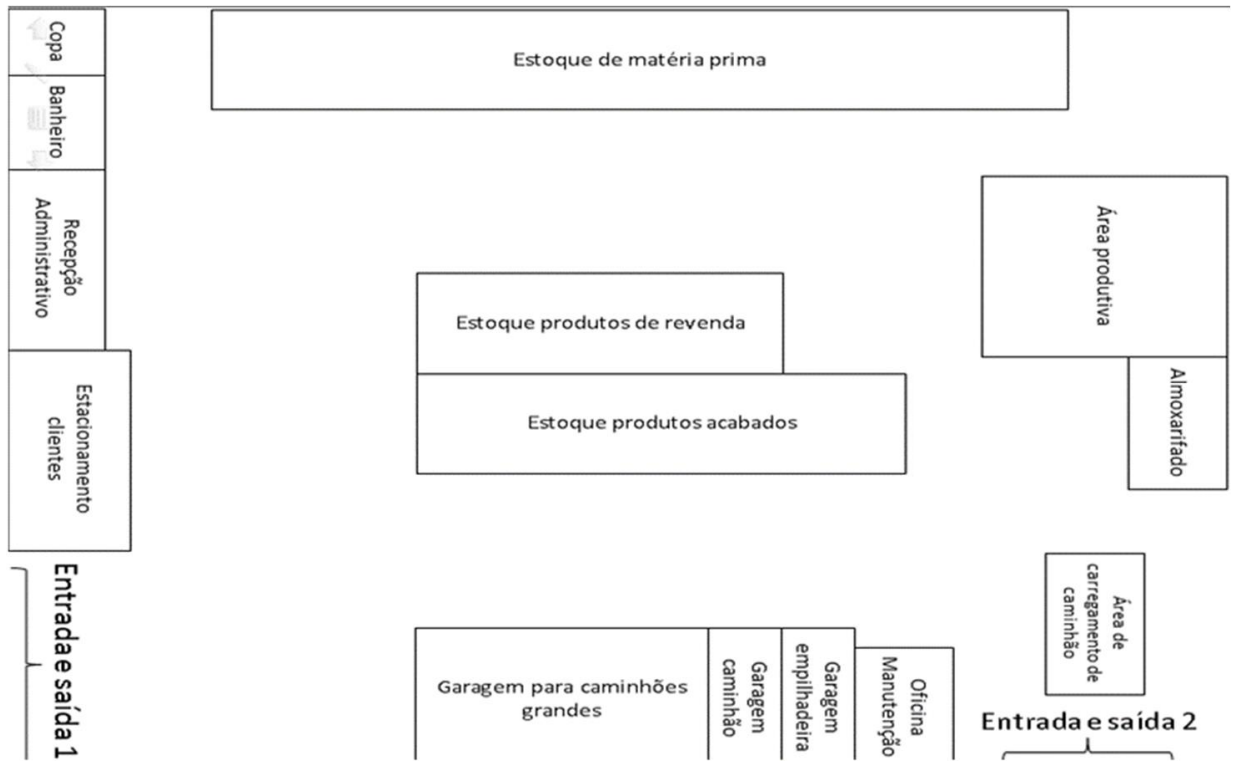

Figura 5 - Proposta 2 de Layout para a empresa de Artefatos de Concreto. 


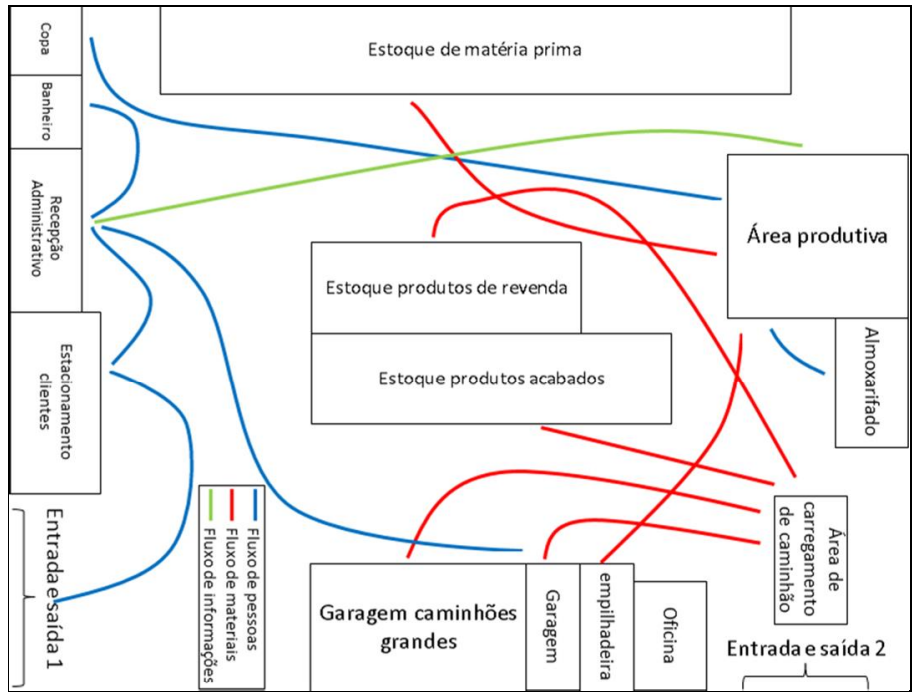

\subsection{Desenvolvimento da proposta 3}

Primeiramente no layout 3, a construção existente no local hoje deverá ser demolida para a realização da nova proposta. Na figura 7 está identificada esta proposta de layout e na figura 8 é apresentado o fluxo de pessoas (linha azul), o fluxo de materiais (linha vermelha) e o fluxo de informações (linha verde). Já na figura 9 além do espaço que será utilizado para a realização das operações e movimentações na proposta de layout 3 .

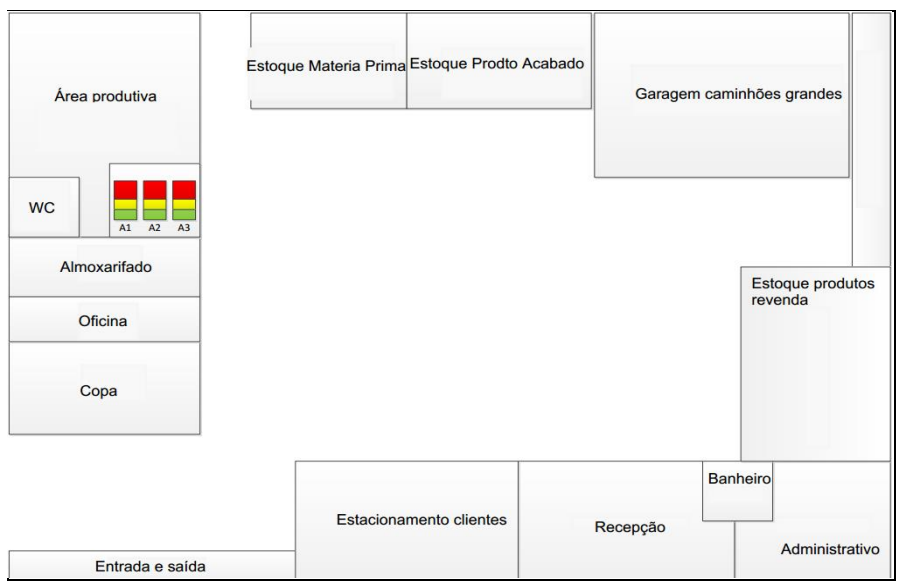

Figura 7 - Proposta 3 de Layout para a empresa de Artefatos de Concreto.

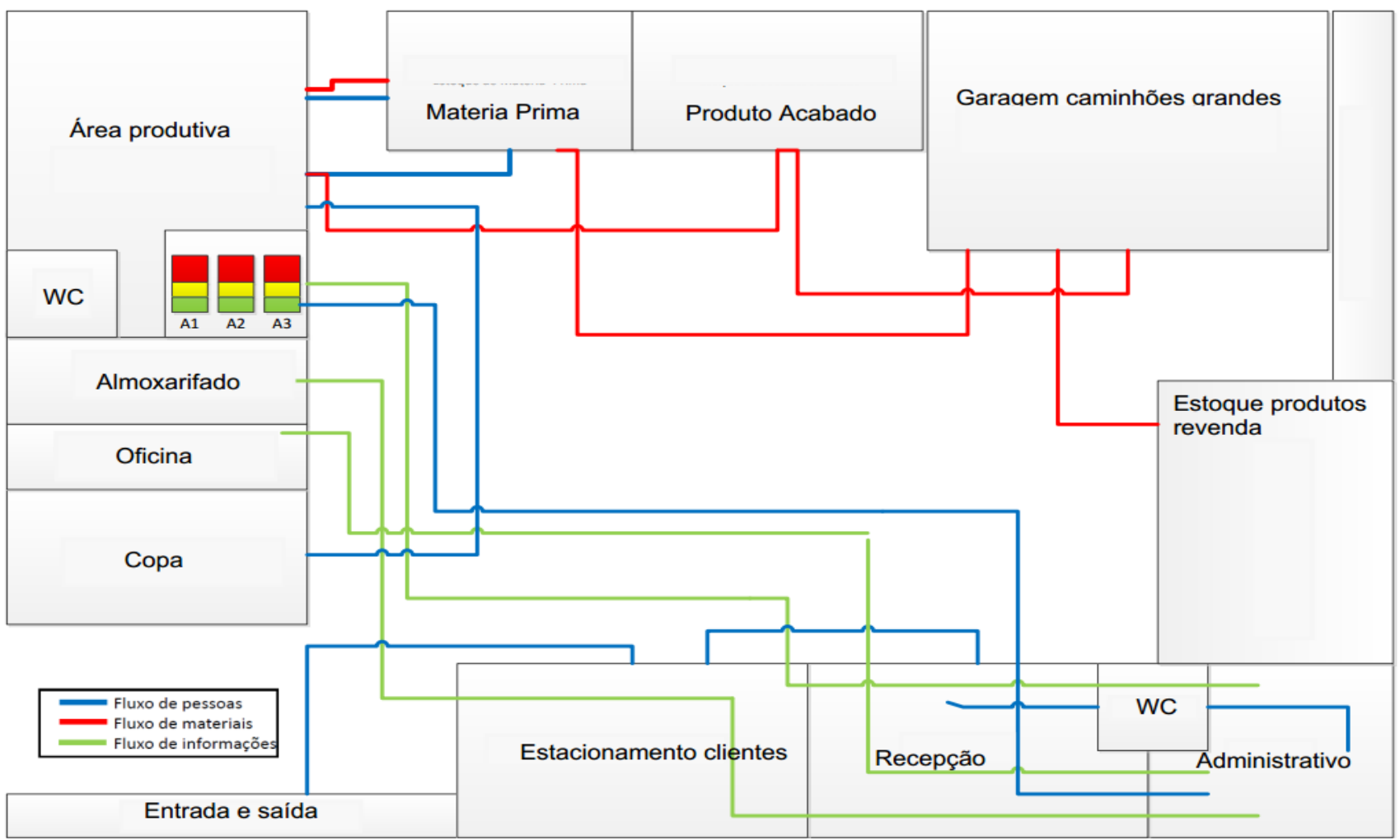

Figura 8 - Análise referente a movimentação de pessoas, materiais e informações na Proposta de Layout 3. 
Na Figura 9 é apresentado o Diagrama de Uso de Espaços referente a Proposta de Layout 3. Nesta pode-se observar o espaço que será utilizado para a realização das operações e movimentações (áreas em destaque).

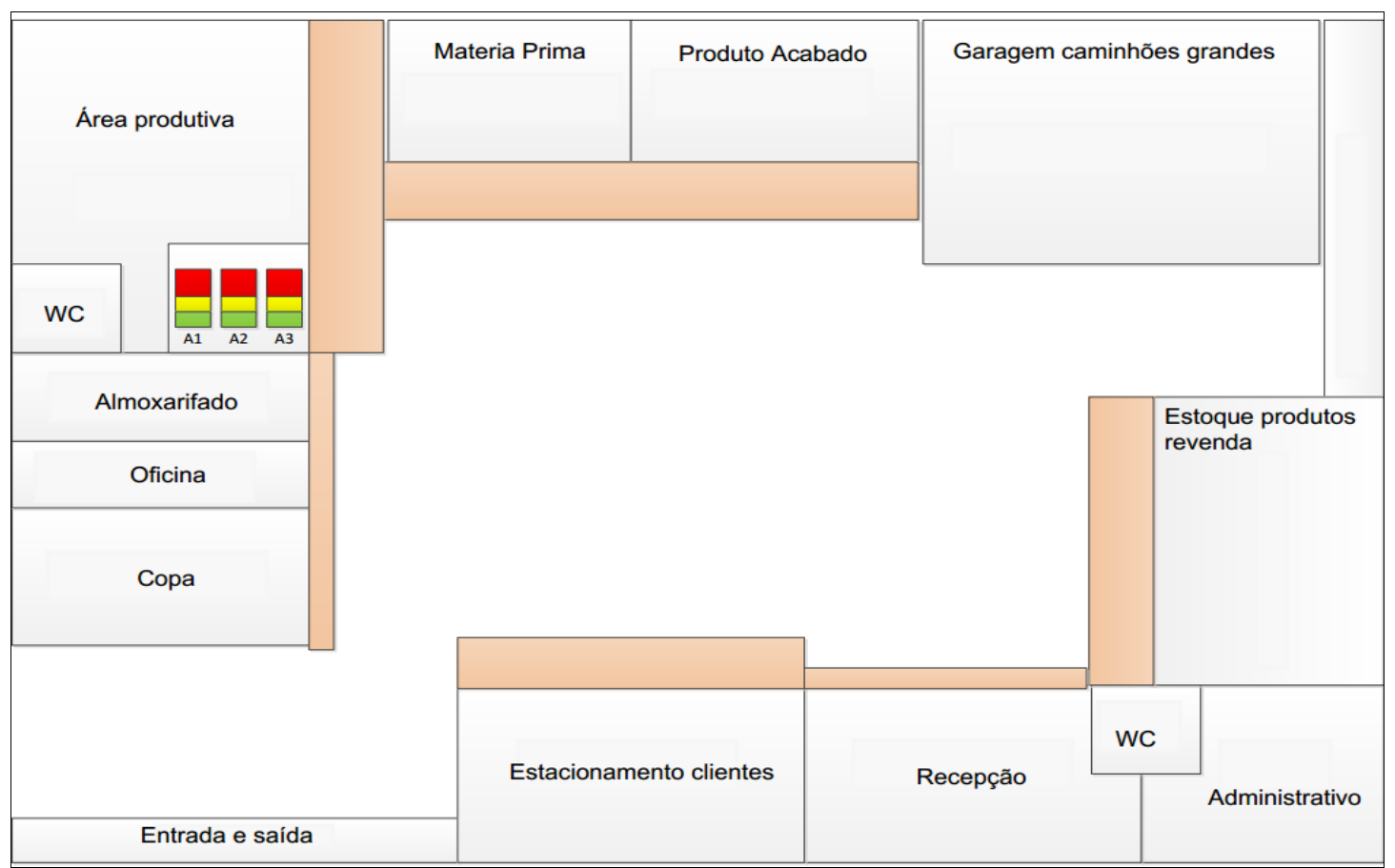

Figura 9 - Diagrama de Uso de Espaços referente a Proposta de Layout 3.

Dentre as diversas vantagens presentes na proposta de layout 3 pode-se destacar: grande área para movimentação interna na empresa; divisão de áreas como produtiva, movimentação e atendimento ao cliente e gerenciamento; sistema de controle de produção, todavia algumas desvantagens da proposta de layout 3 são: elevado custo para a construção; dificuldade para a implantação do sistema kanban; copa próxima a entrada e saída.

\subsection{Escolher alternativas}

As atividades de Escolha das alternativas de layout consistem em duas etapas:

- Avaliação do desempenho individual;

- Seleção do layout.

A comparação entre as alternativas foi realizada com base em seis tópicos (Ver tabela 5), o qual se julgou de maior importância. Cada um desses tópicos recebeu um peso diferente, para que pudesse ponderar os resultados e se obter um comparativo eficiente entre os layouts propostos, conforme indicado na Tabela 5.
Tabela 5 - Matriz de seleção das propostas de layout fabril.

\begin{tabular}{|c|c|c|c|c|}
\hline \multirow{2}{*}{ Características } & \multirow{2}{*}{$\begin{array}{l}0 \\
\vdots \\
2\end{array}$} & \multicolumn{3}{|c|}{ Propostas } \\
\hline & & 1 & 2 & 3 \\
\hline Movimentação de materiais & 5 & - & \multirow{9}{*}{ 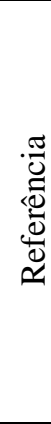 } & - \\
\hline Adequação ao ambiente disponível & 3 & - & & 0 \\
\hline Custo & 4 & 0 & & + \\
\hline Acessibilidade para os clientes & 4 & - & & 0 \\
\hline Espaço de manobras para veículos & 5 & - & & - \\
\hline Uso do espaço & 3 & + & & - \\
\hline & $x+$ & 1 & & 1 \\
\hline & $x-$ & -4 & & -3 \\
\hline \multicolumn{2}{|c|}{ Xtotal } & -3 & & -2 \\
\hline \multicolumn{2}{|c|}{$\times$ Ponderado } & -14 & 0 & -9 \\
\hline
\end{tabular}

Com o desenvolvimento desta análise pode-se observar que a melhor opção de layout seria a Proposta 2, a qual coincidentemente foi à proposta escolhida como referência na matriz de seleção utilizada durante a avaliação. Por fim pode-se realizar o detalhamento do layout final para a Proposta 2, este é apresentado na Figura 10. 


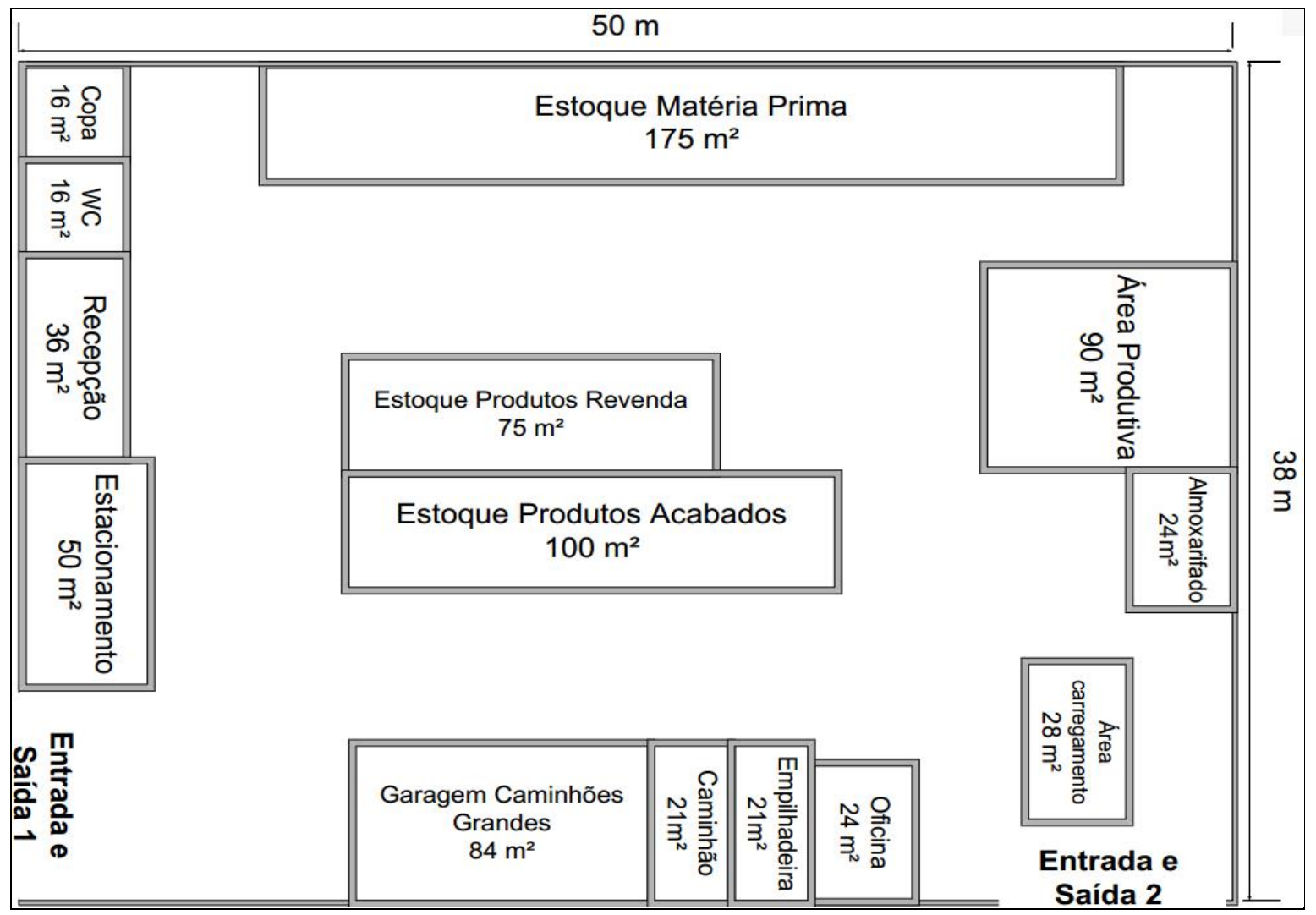

Figura 10 - Layout Detalhado referente a proposta selecionada para a empresa de Artefatos de Concreto.

\section{CONCLUSÃO}

A metodologia desenvolvida por Neumann e Scalice (2015), para realização de um projeto de layout fabril é subdividido em: Projeto Informacional, Conceitual e Detalhado, visto que neste artigo somente foi realizado o Projeto Conceitual, devido à complexidade da realização do projeto completo nesta publicação.

Como o objetivo geral deste trabalho é desenvolver o projeto conceitual do layout para uma empresa de Artefatos de Concreto, foram realizadas tais atividades como: definição das unidades de planejamento de espaços, resumo das unidades de planejamento de espaços, diagrama de afinidades entre UPEôs, diagrama de configuração, realização de layouts, análises de movimentações de pessoas, materiais e informações para cada layout proposto, diagrama de uso de espaço para os layouts propostos, além da matriz de seleção das propostas de layout. Estas atividades auxiliaram no desenvolvimento do projeto de layout para uma empresa classificada com baixa complexidade, visto que a empresa possui como necessidade principal a adequação da sua área fabril buscando melhor utilização do espaço bem como ganho na produtividade.

O layout com as melhores características, que atendem as especificações para os objetivos da empresa, foi selecionado como alternativa viável de implantação. Este atende requisitos de movimentação de materiais, garantindo os melhores tempos produtivos, adequando o ambiente as atividades desenvolvidas em cada setor. Isto faz um alinhamento entre custo e acessibilidade, refletindo diretamente na produtividade.

Como sugestão de trabalhos futuros para este projeto, pode-se considerar o desenvolvimento das demais etapas da fase de Projeto Detalhado referente à metodologia proposta por Neumann e Scalice (2015), a qual consiste em: consolidar layout, otimizar layout e detalhar postos de trabalho.

R EFERÊ N C I A S

BONECHER, D. et al. Proposta de alteração do layout de um centro de distribuição de produtos alimentícios utilizando simulação de cenários e o método de processo de análise hierárquica (AHP). Revista Gestão da Produção Operações e Sistemas, [S.1.], v. 15, n. 2, p. 58, jun. 2020. ISSN 1984-2430. Disponível em: <https://revista.feb.unesp.br/index.php/gepros/article/vi ew/2430>. Acesso em: 22 jun. 2020. doi: https://doi.org/10.15675/gepros.v15i2.2430.

FIGUEIREDO, L.H.W. Aplicação dos tipos de layout: uma análise de produção científica. Brasília: 2016

FLICK, U. Uma Introdução à Pesquisa Qualitativa. 2. ed. São Paulo: Artmed, 2009.

GURGEL, F. A. Logística Industrial. São Paulo: Atlas, 2008.

LIMA, C. R. Projeto de novo layout: Estudo de caso em uma indústria de confecção. Brasília: 2016.

MOREIRA, D. A. Administração da Produção e Operações. São 
Paulo: Pioneira, 2004.

MOURA, C. R.; BORGES, W. J.; MEINCHEIM, E.; G. C. CARLINI.; OLIVEIRA, L. Aplicação do Método SLP no desenvolvimento de um layout otimizado em uma empresa têxtil. ScientiaTec, v. 6 n. 2. 2019. doi: https://doi.org/10.35819/scientiatec.v6i2.3507

NEUMANN, C; SCALICE, R. K. Projeto de fábrica e layout. 1. ed. Rio de Janeiro: Elsevier, 2015.
OLIVEIRA, I. M. D.; da PAZ, C. C.; da SILVA, A. M., \& de PAULA FERREIRA, W. Balanceamento de linha e arranjo físico: estudo de caso em uma linha de produção de cabines para máquinas de construção. Exacta, v. 15 n. 1, 101-110. $2017 . \quad$ doi: https://doi.org/10.5585/exactaep.v15n1.6697 